\title{
Absence of meaningful neurocognitive recovery in comatose patients with primary central nervous system lymphoma despite an effective response to chemotherapy: Case reports
}

\author{
RYAN MAO HENG LIM ${ }^{1}$ and JASON YONGSHENG CHAN ${ }^{2-5}$ \\ ${ }^{1}$ Yong Loo Lin School of Medicine, National University of Singapore, Singapore 117597; ${ }^{2}$ Division of Medical Oncology, \\ National Cancer Centre Singapore, Singapore 169610; ${ }^{3}$ SingHealth Duke-NUS Blood Cancer Centre; \\ ${ }^{4}$ Duke-NUS Medical School, Singapore $169857 ;{ }^{5}$ Cancer Science Institute of Singapore, \\ National University of Singapore, Singapore 117599, Republic of Singapore
}

Received September 7, 2020; Accepted December 23, 2020

DOI: $10.3892 / \mathrm{mco} .2021 .2206$

\begin{abstract}
Primary central nervous system lymphoma (PCNSL) is a rare type of non-Hodgkin's lymphoma that occurs in patients who are elderly and immunocompromised. The most common treatment for PCNSL is high-dose methotrexate-based chemotherapy. Studies have suggested that the radiological response to high-dose methotrexate-based chemotherapy is associated with improved neurocognitive ability that remains stable upon follow-up. However, no study involving patients with an extremely poor neurological status before chemotherapy initiation has been reported, and the neurological prognosis of this group of patients remains unknown. The current case study described 3 patients with PCNSL diagnosed via biopsy who had comatose neurological states due to disease progression prior to treatment. All patients were treated with high-dose methotrexate-based chemotherapy. However, although effective radiological responses to treatment were achieved, no meaningful neurological or cognitive recovery was documented. Patients with PCNSL exhibiting a baseline comatose state have a poor neurological prognosis even with an effective tumour response to chemotherapy. Therefore, rapid detection and prompt treatment are crucial in patients with this disease.
\end{abstract}

Correspondence to: Dr Jason Yongsheng Chan, Division of Medical Oncology, National Cancer Centre Singapore, 11 Hospital Drive, Singapore 169610, Republic of Singapore

E-mail: jason.chan.y.s@nccs.com.sg

Abbreviations: PCNSL, primary CNS lymphoma; MMSE, mini-mental state examination; WBRT, whole-brain radiotherapy; GCS, Glasgow Coma Scale; DLBCL, diffuse large B-cell lymphoma; GCB, germinal centre B-cell like

Key words: primary central nervous system lymphoma, chemotherapy, Glasgow Coma Scale, neurocognition, prognosis, case report

\section{Introduction}

Primary central nervous system lymphoma (PCNSL) is a rare form of non-Hodgkin's lymphoma that is usually confined to the brain, leptomeninges, spine, cerebrospinal fluid and eyes without evidence of systemic spread (1). PCNSL represents approximately $4 \%$ of all newly diagnosed central nervous system (CNS) tumours $(2,3)$, and most seem to be of late or post-germinal centre B-cell origin $(4,5)$. Immunodeficiency due to congenital immunodeficiency syndromes such as ataxia-telangiectasia and Wiskott-Aldrich syndrome, as well as secondary causes such as acquired immunodeficiency syndrome and iatrogenic immunosuppression for transplant procedures, has been implicated in the development of PCNSL (6).

The prognosis of PCNSL has improved substantially in recent years, particularly in immunocompetent patients (7). However, treatment of PCSNL may cause neurotoxicity and compromise health-related quality of life. Studies have suggested that patients treated with combined high-dose methotrexate chemotherapy and consolidation whole-brain radiotherapy (WBRT) in the treatment of PCNSL develop worse neurotoxicity and cognitive dysfunction than those treated with chemotherapy alone (8-12). Thus, consolidation WBRT is often withheld unless necessary, particularly in elderly patients (13). Conversely, cognition, as measured by mini-mental state examination (MMSE) scores, frequently improves following the successful treatment of PCNSL with chemotherapy with or without immunotherapy (rituximab) and remains stable on follow-up (10,14-16). However, currently, no study has described the neurological prognosis of PCNSL patients with a very poor neurocognitive function at baseline.

In this study, we reviewed the cases of 3 patients with neuroimaging- and biopsy-proven PCNSL who had baseline comatose neurological states at presentation (Table I). All 3 were treated with high-dose methotrexate-based chemotherapy only without WBRT and achieved either a partial or complete response to treatment, as assessed using International PCNSL Collaborative Group criteria (17). 


\section{Case reports}

Case 1. A 73-year-old Chinese man presented with worsening lethargy and drowsiness, together with behavioural changes for 1 month. He had a medical history of hypertension, diabetes mellitus, hyperlipidaemia and ischaemic heart disease. During his initial admission, magnetic resonance imaging (MRI) of the brain revealed multiple foci of abnormal enhancement with low to heterogeneous T2 signals in a periventricular distribution, including the ependymal margins of both lateral ventricles, infundibular recess of the 3 rd ventricle and pituitary stalk. These findings were highly suggestive of PCNSL. Despite these findings, the patient declined further work-up and was discharged against medical advice. He was subsequently re-admitted a month later for progressive drowsiness and fever. Neurological examination revealed a severely depressed level of consciousness with a Glasgow Coma Scale (GCS) of E3V1M1. Imaging of the brain showed interval disease progression and obstructive hydrocephalus. The patient subsequently underwent an external ventricular drain insertion, followed by stereotactic biopsy of the right caudate region two weeks later.

Histological evaluation confirmed diffuse large B-cell lymphoma (DLBCL), non-germinal centre B-cell like (non-GCB) subtype. He was administered dexamethasone, procarbazine, vincristine and high-dose methotrexate $\left(2.5 \mathrm{~g} / \mathrm{m}^{2}\right)(18)$. At the point of treatment initiation, the GCS remained poor at E2V2M1. The time from the initial presentation to time of treatment initiation was 2 months. Because chemotherapy was complicated by repeated infective episodes, including pyelonephritis, colitis and pneumonia, only 4 cycles were administered (up to week 8). Brain MRI performed at the end of treatment showed no residual tumour or interval new tumour, indicating a complete response (Fig. 1A).

Despite the complete response of the lymphoma to chemotherapy, the patient's neurocognitive status did not improve and the GCS remained poor at E2V2M1. The patient eventually died of relapsed disease 8 months later.

Case 2. A 42-year-old Bruneian man with no significant medical history initially presented with diplopia and was referred to the National Cancer Centre, Singapore, for suspected PCNSL on preliminary brain imaging. Physical examination revealed a GCS of E3V4M6, cranial nerve III, IV and VI palsy bilaterally, a fixed left pupil and extremely poor visual acuity with inability to visualize light bilaterally. Mild left hemiparesis was also evident. Brain MRI revealed homogeneously enhancing lesions in the optic chiasma, optic nerves and tract, midbrain and pons, and hypothalamus.

Stereotactic biopsy of the left suprasellar lesion confirmed DLBCL, non-GCB type. Unfortunately, the patient became progressively drowsier because of the interval enlargement of the known suprasellar mass associated with an increasing mass effect and worsening hydrocephalus. The patient subsequently underwent ventriculoperitoneal shunt insertion and tracheostomy, while his GCS continued to deteriorate to E1VTM1. He was administered high-dose methotrexate-based chemotherapy for 5 cycles based on the protocol by Shah et al (rituximab, vincristine, procarbazine and methotrexate $\left.2.5 \mathrm{~g} / \mathrm{m}^{2}\right)(19)$. The time from the initial presentation to the start of treatment was 6 months. Post-treatment brain MRI showed a stable hypothalamus lesion and marked improvement in the lesions in the right temporal lobe, bilateral basal ganglia and brainstem, indicating an effective partial response to treatment (Fig. 1B).

Despite the overall response to treatment, the patient did not achieve significant improvement in cognition or physical function. His post-treatment GCS remained low at E2V1M1. Thereafter, he was managed with best supportive care alone at a hospice.

Case 3. A 68-year-old Chinese man with a significant medical history of hypertension, hyperlipidaemia and stroke presented with an unsteady gait with frequent falls, impairment of semantic memory and slow speech. His physical examination was unremarkable, and he had no gross neurological deficits. His GCS was E4V4M6. Brain MRI showed lobulated semi-confluent enhancing lesions at the bilateral periventricular regions involving the corpus callosum and corona radiata.

Stereotactic biopsy of the left frontal periventricular region revealed DLBCL, GCB type. Post operatively, he was administered levetiracetam and dexamethasone because he had mild fasciculations of his right thigh and twitching of his left biceps suggestive of a provoked seizure. He was intubated when his GCS subsequently deteriorated to E1V1M1, following which a tracheostomy was performed. Subsequently, he was administered high-dose methotrexate-based chemotherapy for 5 cycles including rituximab, vincristine and methotrexate $\left(2.5 \mathrm{~g} / \mathrm{m}^{2}\right)$ but not procarbazine. Post-treatment brain MRI revealed complete resolution of the lesions along the periventricular region and corpus callosum, indicating a complete response to treatment (Fig. 1C).

Similar to cases 1 and 2, despite an effective tumour response to chemotherapy, he remained in a comatose state with a GCS of 3 and died 8 months later.

\section{Discussion}

We described the cases of 3 patients with extremely poor neurological statuses before treatment initiation. All 3 patients did not achieve meaningful neurological recovery despite an effective tumour response to chemotherapy, as evidenced by the low post-treatment GCS of 5, 4 and 3 for cases 1, 2 and 3, respectively. These findings contrast those of earlier studies that reported an improvement in cognitive function following the successful treatment of PCNSL $(10,14-16)$. Although these cohorts generally comprise patients with grossly intact neurological and cognitive function (median MMSE range, 22-23), our study is novel because the 3 patients had extremely poor neurocognitive function at baseline with a GCS score of 5 or below before treatment initiation.

The GCS decreased in all 3 patients in the short time frame between the initial presentation and start of treatment, ranging from 12 days to 6 months. In case 1, treatment was delayed because the patient had initially requested for discharge against medical advice, whereas the delay in case 2 was due to the patient being referred from an overseas hospital. In case 3 , treatment was promptly commenced. Thus, PCNSL is an aggressive disease with an unpredictable clinical course. A sharp decrease in the GCS representing disease progression 


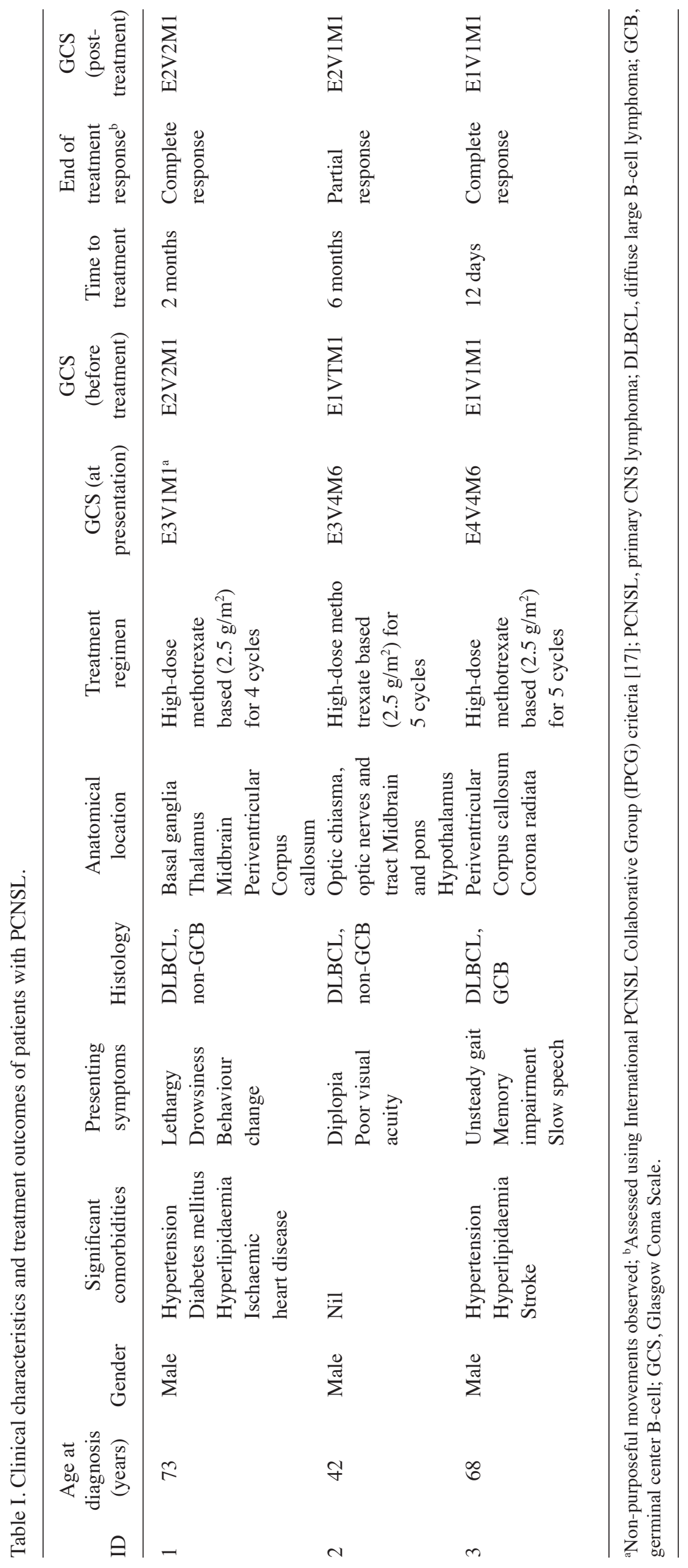


A

Case 1

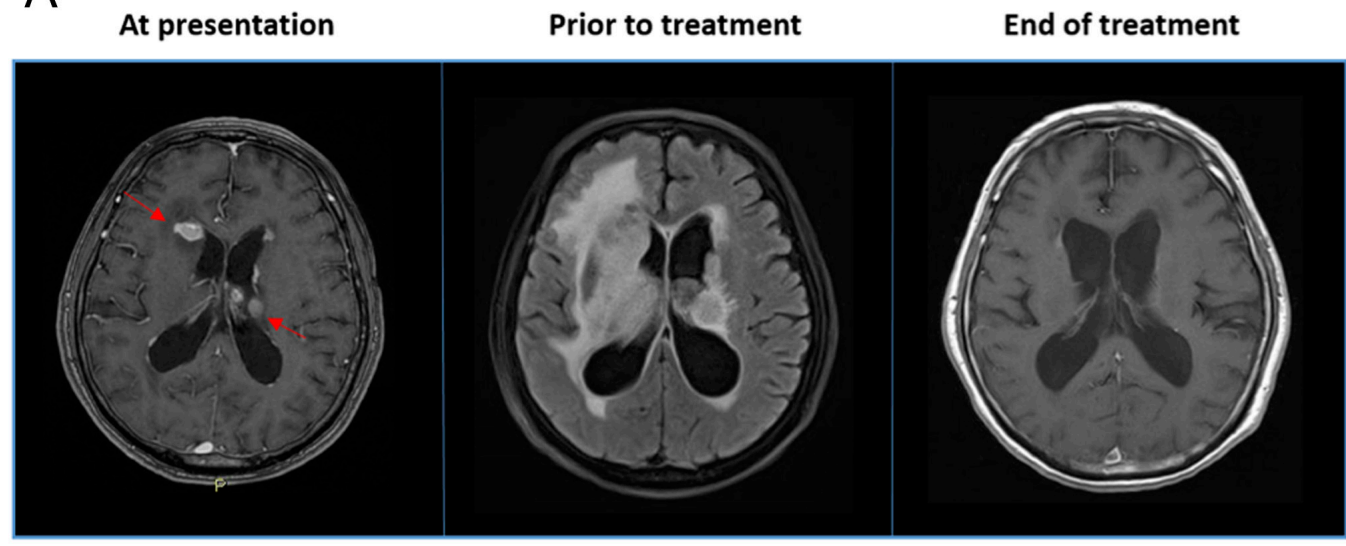

B

Case 2
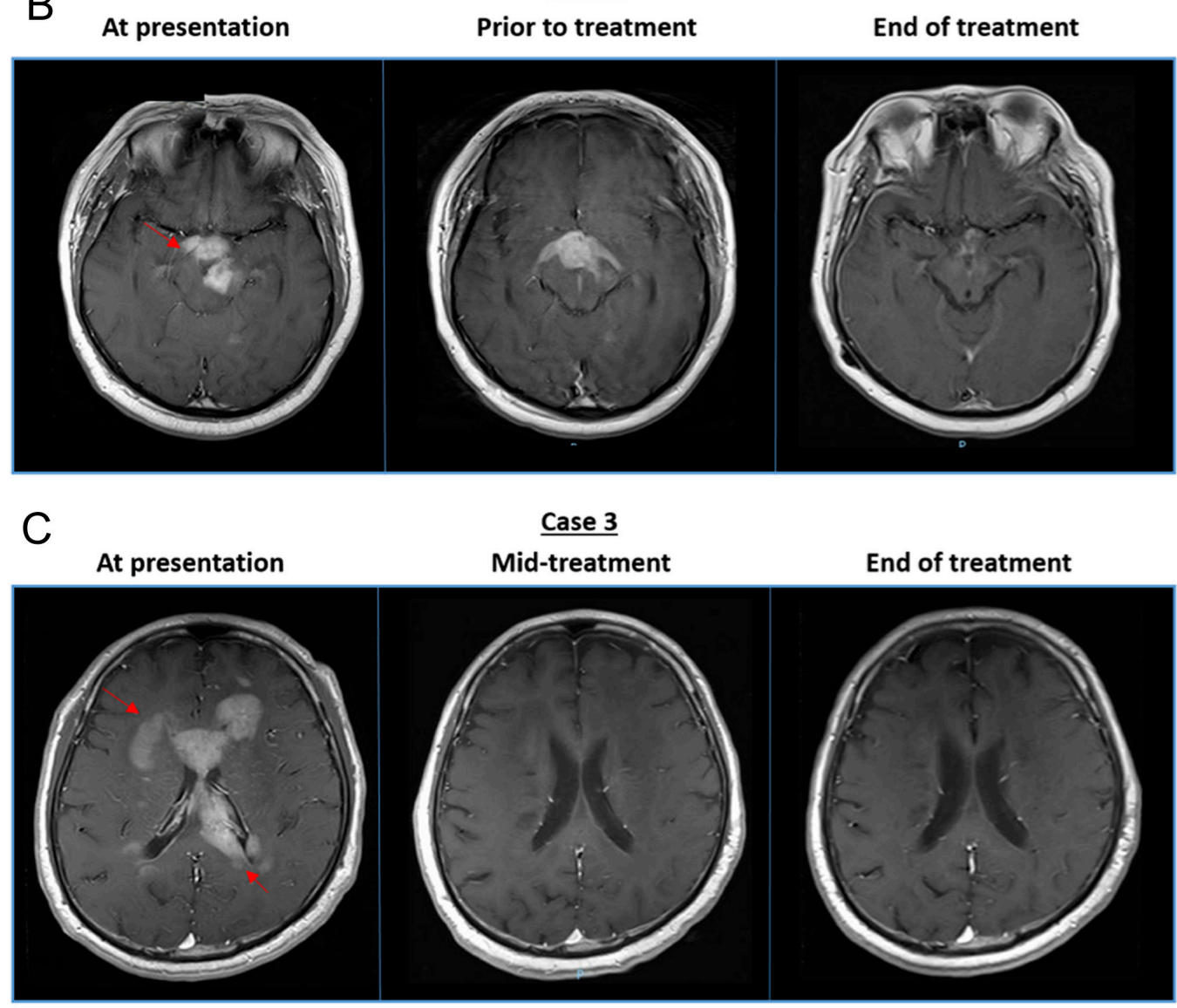

Figure 1. T1-weighted MRI images reveal the anatomical involvement of PCNSL at various junctures of treatment. (A) Complete responses to chemotherapy were observed in case 1, (B) partial response to chemotherapy was observed in case 2 and (C) complete responses to chemotherapy were observed in case 3 . The red arrows indicate the initial sites of disease. PCNSL, primary CNS lymphoma.

of PCNSL may indicate a poor neurological prognosis even if the tumour responds well to chemotherapy. In such a scenario, best supportive care focusing on the quality of life may be considered and weighed carefully against aggressive chemotherapy with curative intent.

Although cases 1 and 3 involved elderly Chinese patients older than 65 years, case 2 involved a young Bruneian man aged only 42 years at diagnosis. Despite the differences in both age and race, all 3 patients had similarly poor neurological outcomes at the end of chemotherapy treatment. This supports the hypothesis that a poor neurocognitive status before treatment is a poor prognostic indicator for the post-treatment neurological outcome across various demographical factors such and age and race.

In conclusion, the neurocognitive status of PCNSL patients can deteriorate quickly, indicating dismal outcomes. Patients with severe neurocognitive compromise may have a poor neurological prognosis despite an effective response to treatment. Further validation studies should be conducted to examine the neurological prognosis of PCNSL patients with poor neurological function at baseline who were treated successfully with chemotherapy, as well as to determine the 
possible causes of and prevent poor neurological status in these patients. Our study suggests that administering early treatment in PCNSL patients and avoiding unnecessary delays are necessary to achieve optimal neurocognitive recovery.

\section{Acknowledgements}

Not applicable.

\section{Funding}

This work was supported by the Singapore Ministry of Health's National Medical Research Council of Singapore (grant no. NMRC/FLWSHP/054/2017-00), SHF-Foundation (grant no. SHF/FG653P/2017) and SingHealth Duke-NUS Academic Medical Centre and Oncology ACP (grant no. 08-FY2017/P1/14-A28).

\section{Availability of data and materials}

Data sharing is not applicable to this article, as no datasets were generated or analysed during the present study.

\section{Authors' contributions}

RMHL and JYC conceptualized the study and wrote the manuscript. RMHL acquired, analysed and interpreted the data. JYC enrolled the study patients, obtained their consent and treated them. Both authors have confirmed the authenticity of all raw data, as well as read and approved the final manuscript.

\section{Ethics approval and consent to participate}

The present study was approved by the Singhealth Centralised Institutional Review Board (CIRB 2018/3084). Written informed consent was obtained from all the participants and/or their legal guardians.

\section{Patient consent for publication}

Written informed consent was obtained from the patients for the publication of this case report and any accompanying images.

\section{Competing interests}

The authors declare that they have no competing interests.

\section{References}

1. Grommes C and DeAngelis LM: Primary CNS lymphoma. J Clin Oncol 35: 2410-2418, 2017.

2. Villano JL, Koshy M, Shaikh H, Dolecek TA and McCarthy BJ: Age, gender, and racial differences in incidence and survival in primary CNS lymphoma. Br J Cancer 105: 1414-1418, 2011.

3. Hoffman S, Propp JM and McCarthy BJ: Temporal trends in incidence of primary brain tumors in the United States, 1985-1999. Neuro Oncol 8: 27-37, 2006.
4. Montesinos-Rongen M, Brunn A, Bentink S, Basso K, Lim WK, Klapper W, Schaller C, Reifenberger G, Rubenstein J, Wiestler OD, et al: Gene expression profiling suggests primary central nervous system lymphomas to be derived from a late germinal center B cell. Leukemia 22: 400-405, 2008.

5. Camilleri-Broët $S$, Criniè E, Broët $P$, Delwail V, Mokhtari K, Moreau A, Kujas M, Raphaël M, Iraqi W, Sautès-Fridman C, et al: Auniform activated B-cell-like immunophenotype might explain the poor prognosis of primary central nervous system lymphomas: Analysis of 83 cases. Blood 107: 190-196, 2006.

6. Bhagavathi S and Wilson JD: Primary central nervous system lymphoma. Arch Pathol Lab Med 132: 1830-1834, 2008.

7. Shiels MS, Pfeiffer RM, Besson C, Clarke CA, Morton LM, Nogueira L, Pawlish K, Yanik EL, Suneja G and Engels EA: Trends in primary central nervous system lymphoma incidence and survival in the U.S. Br J Haematol 174: 417-424, 2016.

8. Correa DD, DeAngelis LM, Shi W, Thaler H, Glass A and Abrey LE: Cognitive functions in survivors of primary central nervous system lymphoma. Neurology 62: 548-555, 2004

9. Batchelor T and Loeffler JS: Primary CNS lymphoma. J Clin Oncol 24: 1281-1288, 2006.

10. van der Meulen M, Dirven L, Habets EJJ, van den Bent MJ, Taphoorn MJB and Bromberg JEC: Cognitive functioning and health-related quality of life in patients with newly diagnosed primary CNS lymphoma: A systematic review. Lancet Oncol 19: e407-e418, 2018.

11. Doolittle ND, Korfel A, Lubow MA, Schorb E, Schlegel U, Rogowski S, Fu R, Dósa E, Illerhaus G, Kraemer DF, et al: Long-term cognitive function, neuroimaging, and quality of life in primary CNS lymphoma. Neurology 81: 84-92, 2013.

12. Thiel E, Korfel A, Martus P, Kanz L, Griesinger F, Rauch M, Röth A, Hertenstein B, von Toll T, Hundsberger T, et al: High-dose methotrexate with or without whole brain radiotherapy for primary CNS lymphoma (G-PCNSL-SG-1): A phase 3, randomised, non-inferiority trial. Lancet Oncol 11: 1036-1047, 2010.

13. Sierra del Rio M, Rousseau A, Soussain C, Ricard D and Hoang-Xuan K: Primary CNS lymphoma in immunocompetent patients. Oncologist 14: 526-539, 2009.

14. Fritsch K, Kasenda B, Schorb E, Hau P, Bloehdorn J, Möhle R, Löw S, Binder M, Atta J, Keller U, et al: High-dose methotrexate-based immuno-chemotherapy for elderly primary CNS lymphoma patients (PRIMAIN study). Leukemia 31: 846-852, 2017.

15. Batchelor T, Carson K, O'Neill A, Grossman SA, Alavi J, New P, Hochberg F and Priet R: Treatment of pimary CNS lymphoma with methotrexate and deferred radiotherapy: A report of NABTT 96-07. J Clin Oncol 21: 1044-1049, 2003.

16. Hoang-Xuan K, Taillandier L, Chinot O, Soubeyran P, Bogdhan U, Hildebrand J, Frenay M, De Beule N, Delattre JY and Baron B; European Organization for Research and Treatment of Cancer Brain Tumor Group: Chemotherapy alone as initial treatment for primary CNS lymphoma in patients older than 60 years: A multicenter phase II study (26952) of the European Organization for Research and Treatment of Cancer Brain Tumor Group. J Clin Oncol 21: 2726-2731,2003.

17. Abrey LE, Batchelor TT, Ferreri AJ, Gospodarowicz M, Pulczynski EJ, Zucca E, Smith JR, Korfel A, Soussain C, DeAngelis LM, et al: Report of an international workshop to standardize baseline evaluation and response criteria for primary CNS lymphoma. J Clin Oncol 23: 5034-5043, 2005.

18. DeAngelis LM, Seiferheld W, Schold SC, Fisher B and Schultz CJ; Radiation Therapy Oncology Group Study 93-10: Combination chemotherapy and radiotherapy for primary central nervous system lymphoma: Radiation Therapy Oncology Group Study 93-10. J Clin Oncol 20: 4643-4648, 2002.

19. Shah GD, Yahalom J, Correa DD, Lai RK, Raizer JJ, Schiff D, LaRocca R, Grant B, DeAngelis LM and Abrey LE: Combined immunochemotherapy with reduced whole-brain radiotherapy for newly diagnosed primary CNS lymphoma. J Clin Oncol 25: 4730-4735, 2007.

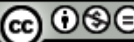

This work is licensed under a Creative Commons Attribution-NonCommercial-NoDerivatives 4.0 International (CC BY-NC-ND 4.0) License. 